\title{
Reply to the Letter on "Closeness to friends explains age differences in positive emotional experience during the lockdown period of COVID-19 pandemic"
}

\author{
Elena Cavallini ${ }^{1}$ (1) - Alessia Rosi ${ }^{1}$. Floris Tijmen van Vugt ${ }^{2} \cdot$ Irene Ceccato $^{3} \cdot$ Filippo Rapisarda $^{4} \cdot$ Martine Vallarino $^{1}$. \\ Luca Ronchi ${ }^{1} \cdot$ Tomaso Vecchi $^{1,5} \cdot$ Serena Lecce ${ }^{1}$
}

Received: 3 August 2021 / Accepted: 24 August 2021 / Published online: 8 September 2021

(c) The Author(s), under exclusive licence to Springer Nature Switzerland AG 2021

\section{Dear Editor,}

We would like to thank Dr. Rujittika Mungmunpuntipantip and Prof. Viroj Wiwanitkit for their interest in our manuscript "Closeness to friends explains age differences in positive emotional experience during the lockdown period of COVID-19 pandemic" [1]. In our article, we investigated age differences in positive and negative emotional experiences and whether the perception of closeness to family/friends and the engagement in daily activities during pandemic explained such age-related differences. We found that the perception of more closeness to friends, but not the engagement in daily activities, explained differences in positive emotional experience.

Dr. Rujittika Mungmunpuntipantip and Prof. Viroj Wiwanitkit questioned about the difficulties in using social network in some areas, such as those in Asia, because of limited infrastructure [2]. We would like to specify that we used the expression "social network" referring to its original meaning that is social contacts with friends, family, or colleagues. We asked participants to report their perception of

Elena Cavallini and Alessia Rosi equally contributed as co-first authors.

Elena Cavallini

ecava@unipv.it

1 Department of Brain and Behavioral Sciences, University of Pavia, Piazza Botta 6, 27100 Pavia, Italy

2 Department of Psychology, University of Montreal, Montreal, QC, Canada

3 Deparment of Neuroscience, Imaging and Clinical Sciences, University G. d'Annunzio of Chieti-Pescara, Chieti, Italy

4 Sociosfera ONLUS SCS, Seregno, Italy

5 Cognitive Psychology Unit, IRCCS Mondino Foundation, Pavia, Italy closeness and, given its critical role in promoting emotional wellbeing, especially in older participants and according to the Socioemotional Selectivity Theory [3, 4], we suggest increasing the closeness to social network. This is possible through phone calls or video chats, during the pandemic period, or encounters in presence, during the "normal" life. Hence, we do not consider the social network as a dedicated website or another application which allows users to communicate and share information. Instead, we refer to the social network as the different groups of people that individuals know.

Funding This work was supported by funding from the Italian Ministry of University and Research (PRIN 2017 no. 201755TKFE) and from Italian Ministry of Health (Ricerca Corrente 2020) to Tomaso Vecchi. The authors did not receive support from any other organizations for the submitted work.

\section{Declarations}

Conflict of interest On behalf of all authors, the corresponding author states that there is no conflict of interest.

Ethical approval The study was approved by the Ethical Committee of the Department of Brain and Behavioral Sciences of the University of Pavia (no. 46/2020). The study was conducted in accordance with the ethical standards of the institutional and/or national research committee and with the 1964 Declaration of Helsinki and its later amendments or comparable ethical standards.

Consent to participate Informed consent was obtained from all individual participants included in the study. 


\section{References}

1. Cavallini E, Rosi A, van Vugt FT et al (2021) Closeness to friends explains age differences in positive emotional experience during the lockdown period of COVID-19 pandemic. Aging Clin Exp Res. https://doi.org/10.1007/s40520-021-01927-7 (Online ahead of print)

2. Mungmunpuntipantip R, Wiwanitkit V (2021) Closeness to social network and COVID-19. Aging Clin Exp Res. https://doi.org/10. 1007/s40520-021-01958-0 (Online ahead of print)

3. Carstensen LL, Isaacowitz DM, Charles ST (1999) Taking time seriously: a theory of socioemotional selectivity. Am Psychol 54:165-181. https://doi.org/10.1037//0003-066x.54.3.165
4. English T, Carstensen LL (2014) Selective narrowing of social networks across adulthood is associated with improved emotional experience in daily life. Int J Behav Dev 38:195-202. https://doi. org/10.1177/0165025413515404

Publisher's Note Springer Nature remains neutral with regard to jurisdictional claims in published maps and institutional affiliations. 